\title{
Transverse kink oscillations in the presence of twist
}

\author{
J. Terradas ${ }^{1}$ and M. Goossens ${ }^{2}$ \\ 1 Departament de Física, Universitat de les Illes Balears, 07122, Spain \\ e-mail: jaume.terradas@uib.es \\ 2 Centre for Plasma Astrophysics, Department of Mathematics, Celestijnenlaan 200B, Katholieke Universiteit Leuven, 3001 Leuven, \\ Belgium \\ e-mail: Marcel.Goossens@wis. kuleuven.be
}

Received 2 July 2012 / Accepted 10 October 2012

\begin{abstract}
Context. Magnetic twist is thought to play an important role in coronal loops. The effects of magnetic twist on stable magnetohydrodynamic (MHD) waves is poorly understood because they are seldom studied for relevant cases.

Aims. The goal of this work is to study the fingerprints of magnetic twist on stable transverse kink oscillations.

Methods. We numerically calculated the eigenmodes of propagating and standing MHD waves for a model of a loop with magnetic twist. The azimuthal component of the magnetic field was assumed to be small in comparison to the longitudinal component. We did not consider resonantly damped modes or kink instabilities in our analysis.

Results. For a nonconstant twist the frequencies of the MHD wave modes are split, which has important consequences for standing waves. This is different from the degenerated situation for equilibrium models with constant twist, which are characterised by an azimuthal component of the magnetic field that linearly increases with the radial coordinate.

Conclusions. In the presence of twist standing kink solutions are characterised by a change in polarisation of the transverse displacement along the tube. For weak twist, and in the thin tube approximation, the frequency of standing modes is unaltered and the tube oscillates at the kink speed of the corresponding straight tube. The change in polarisation is linearly proportional to the degree of twist. This has implications with regard to observations of kink modes, since the detection of this variation in polarisation can be used as an indirect method to estimate the twist in oscillating loops.
\end{abstract}

Key words. magnetohydrodynamics (MHD) - waves - Sun: corona

\section{Introduction}

It is anticipated that magnetic twist plays a considerable role in the structure of coronal loops. The highly dynamic photosphere and chromosphere can introduce twist in the magnetic field of the solar corona. For example, footpoint rotation and shear motion may result in twisting of coronal loops (see e.g., Brown et al. 2003). A newly emerged magnetic field is also supposed to be twisted during the buoyant evolution through the convection zone, meaning that loops may emerge already twisted (see e.g., Moreno-Insertis \& Emonet 1996; Hood et al. 2009). Twist also considerably contributes to the eruption of many prominences and coronal mass ejections (CMEs; see e.g., Hood \& Priest 1979; Priest et al. 1989).

In the theoretical analysis of magnetohydrodynamic (MHD) waves, the effects of a curved loop axis are usually omitted. The stability of straight tubes with twisted magnetic field has been investigated by e.g., Shafranov (1957), Kruskal et al. (1958), and Suydam (1958). The role of line tying conditions in the context of coronal loops has been studied by e.g., Raadu (1972) while the effect of gas pressure on the kink instability has been analysed by e.g., Giachetti et al. (1977). The combination of both effects has been studied by Hood \& Priest (1979, 1981), Hood et al. (1982), Einaudi \& van Hoven (1983), and Velli et al. (1990). More recently, Zaqarashvili et al. (2010) and Díaz et al. (2011) have considered the stability of twisted magnetic flux tubes with mass flows along the field lines. The main aim of the previous works is determining the instability threshold and calculating the growth rate of kink unstable modes.
Stable propagating MHD waves have been studied previously, but almost always under particular conditions that somehow reduce the mathematical complexity. Often the MHD waves are incompressible and/or the azimuthal component of the magnetic field varies linearly with distance up to a certain position where it drops to zero or all the way up to infinity. The first choice leads to a surface current while the second choice causes magnetic energy to diverge. Early studies were performed by Goossens et al. (1992) and Bennett et al. (1999) in the incompressible regime. The analysis of sausage modes $(m=0)$ has been carried out by Erdélyi \& Fedun (2006, 2007). Kink modes assuming compressible motions have been considered by Erdélyi \& Carter (2006), Carter \& Erdélyi (2008), Erdélyi \& Fedun (2010). In these works standing transverse waves have not been considered and only propagating waves have been analysed.

In particular, Ruderman (2007, see also review by Ruderman \& Erdélyi 2009) studied standing waves and concluded that twist does not affect kink modes, only fluting modes are modified (see also Goossens et al. 1992). This result is due to the particular choice of constant magnetic twist, i.e., twist is independent of the radial coordinate. The conclusions of the work of Ruderman (2007) have some authors to regard twist as unimportant for transverse kink oscillations.

In this paper we study the effect of an azimuthal magnetic field component on the frequencies of MHD waves for an equilibrium model with nonconstant magnetic twist. We are especially interested in the effect of twist on standing kink modes, a 
problem that, to our knowledge, has not been addressed in the context of transverse coronal oscillations (with the exception of standing fluting modes, studied in Ruderman 2007). Thus, our focus is on the fingerprints of twist on transverse kink oscillations but in the stable regime. This might be relevant for interpreting kink oscillations, from the early transverse kink oscillations reported with TRACE (Aschwanden et al. 1999; Nakariakov et al. 1999), to the last AIA observations of transverse oscillating loops (Aschwanden \& Schrijver 2011).

\section{Equilibrium model of the magnetically twisted loop}

The model for the coronal loop that we use in the present paper is a straight cylinder with constant density inside the cylinder and constant density outside the cylinder. Thus, the coronal loop is modelled as a density enhancement characterised by a density contrast $\rho_{\mathrm{i}} / \rho_{\mathrm{e}}$ with $\rho_{\mathrm{i}}$ being the internal density and $\rho_{\mathrm{e}}$ the external density,

$\rho= \begin{cases}\rho_{\mathrm{i}}, & 0 \leq r<R \\ \rho_{\mathrm{e}}, & r>R .\end{cases}$

At $r=R$ the density changes in a discontinuous manner and $R$ is the radius of the loop.

Under coronal conditions it is a good approximation to neglect plasma pressure compared to magnetic pressure. This zero- $\beta$ approximation removes the slow magnetoacoustic waves from the analysis. In the present paper we allow the magnetic twist to be nonconstant. The model starts from the equation of magnetostatic equilibrium

$\frac{\mathrm{d} B^{2}}{\mathrm{~d} r}=-2 \frac{B_{\varphi}^{2}}{r}$

where

$B^{2}=B_{z}^{2}+B_{\varphi}^{2}$

Here the azimuthal component of the magnetic field is prescribed and Eq. (2) is solved for $B_{z}(x)$ with $x=r / R$. The solution is

$B_{z}^{2}(x)=B_{z}^{2}(0)-B_{\varphi}^{2}(x)-2 \int_{0}^{x} \frac{B_{\varphi}^{2}(s)}{s} \mathrm{~d} s$.

The azimuthal component of the field is written as

$B_{\varphi}(x)=A f(x)$,

where $A$ has the dimension of a magnetic field and $f(x)$ is a dimensionless function satisfying the condition $f(0)=0$ or better $f(x) \rightarrow 0$ for $x \rightarrow 0$. If we insert Eq. (5) in (4) we find

$B_{z}^{2}(x)=B_{z}^{2}(0)-A^{2} G(x)$,

$G(x)=f^{2}(x)+2 \int_{0}^{x} \frac{f^{2}(s)}{s} \mathrm{~d} s$.

Notice that $G(0)=0$ and $G(x) \geq 0$.

A particular choice for $f(x)$ used in the present paper is

$f(x)= \begin{cases}0, & 0 \leq x<p \\ (x-p)(q-x), & p \leq x \leq q, \\ 0, & x>q\end{cases}$

where $p$ and $q$ are constants that we can freely choose. The choice $p=0$ is an option and that $q>1$ is also allowed. For this model we have the freedom to have twist inside and also outside the tube. The function $f(x)$ attains its maximal value $f_{\max }=(q-p)^{2} / 4$ at $x_{\mathrm{m}}=(p+q) / 2$. Hence

$B_{\varphi}(x)=\alpha B_{z}(0) \frac{f(x)}{f_{\max }}$.

In order to relate the strength of the azimuthal component $B_{\varphi}$ with respect to the dominant longitudinal component, we introduce $\alpha$ :

$\alpha=\frac{B_{\varphi, \max }}{B_{z}(0)}$,

hence

$A=\frac{\alpha}{f_{\max }} B_{z}(0)$.

For the function $f(x)$ specified by Eq. (8) it follows that

$G(x)= \begin{cases}0, & 0 \leq x<p, \\ >0, & p \leq x \leq q, \\ G(q), & x>q .\end{cases}$

This function is calculated using Eq. (7).

In summary, the equilibrium magnetic field is defined as

$B_{z}=B_{0}, B_{\varphi}=0$,

for $x<p$,

$B_{z}=\sqrt{B_{0}^{2}-A^{2} G(x)}, B_{\varphi}=A(x-p)(x-q)$,

for $p<x<q$, with

$G(x)=(x-p)^{2}(x-q)^{2}+c_{4}\left(x^{4}-p^{4}\right)+c_{3}\left(x^{3}-p^{3}\right)$

$c_{1}=-4 p q(p+q)$,

$c_{2}=(p+q)^{2}+2 p q$,

$c_{3}=-\frac{4}{3}(p+q)$,

$c_{4}=\frac{1}{2}$,

$c_{l}=2 p^{2} q^{2}$,

and

$B_{\varphi}=0, B_{z}=\sqrt{B_{0}^{2}-A^{2} G(q)}$,

for $x>q$.

\section{MHD eigenmodes}

\subsection{MHD equations and numerical method}

The motions superimposed on the equilibrium model given in the previous section are governed by the linearised ideal MHD equations in cylindrical coordinates. These equations can be found in the Appendix. Several terms involving radial derivatives of the equilibrium magnetic field are present in the equations.

In the MHD equations we have performed a Fourier analysis in time, with $\omega$ the frequency, and also in the azimuthal direction, $m$ being the azimuthal wavenumber. For the longitudinal direction (along the tube axis) two situations are studied. The first situation corresponds to fully propagating waves, allowing 
Fourier decomposition in $z, k$ being the longitudinal wavenumber. In this case the equations are solved in the radial coordinate $r$. The second situation corresponds to standing waves with line-tying conditions at the footpoints of the loop. This problem does not allow Fourier analysis in $z$, and the equations are solved in 2D (in $r$ and $z$ ). For the analysis of propagating waves the system of equations reduces in the ideal regime to the HainLüst equation. In general, it is not possible to find analytical solutions to this equation when the twist profile is nonconstant. For this reason the MHD equations are numerically solved using the code PDE2D (Sewell 2005) in the two situations (1D and 2D) explained above. More details about the numerical method can be found in Terradas et al. (2006). In the present paper we avoided possible Alfvénic resonances on purpose, interesting as they might be. In addition, we stayed away from a strong azimuthal field that can cause kink instabilities of the magnetic flux tube. This means that the eigenfrequency $\omega$ is a purely real magnitude in our analysis.

\subsection{Effect of the azimuthal magnetic field on MHD waves}

Since we adopted the zero- $\beta$ approximation, the only characteristic frequency in the system is the local Alfvén frequency $\omega_{\mathrm{A}}$. The effect that the azimuthal component of the magnetic field $B_{\varphi}$ has on the MHD waves can be appreciated from the effect of $B_{\varphi}$ on $\omega_{\mathrm{A}}^{2}$. The square of the Alfvén frequency is defined as

$\omega_{\mathrm{A}}^{2}=\frac{1}{\mu_{0} \rho}\left(\frac{m}{r} B_{\varphi}+k B_{z}\right)^{2}$

This expression can be written as

$\omega_{\mathrm{A}}^{2}=\frac{B_{z}^{2}}{\mu_{0} \rho} \frac{1}{R^{2}}(k R)^{2}\left(1+\frac{m}{\pi} \Phi(r)\right)^{2}$.

The function $\Phi(r)$ is

$\Phi(r)=\frac{\pi}{k R} \frac{B_{\varphi}}{(r / R) B_{z}}=L \frac{B_{\varphi}}{r B_{z}}$,

where we have defined $L$ that $k=\pi / L . \Phi(r)$ is the twist of the magnetic field lines and it is $2 \pi$ times the number of windings of the field around the loop axis over a distance $L$. Equation (23) suggests that even a relatively small $\varphi$-component of the magnetic field in the sense that $\left|B_{\varphi}\right| \ll\left|B_{z}\right|$, might have a relatively important effect on the MHD waves. The relative importance of $B_{\varphi}$ to $B_{z}$ is controlled by the amount of twist of the magnetic field, with the length $L$ being a relevant factor.

The ratio of the contribution to the Alfvén frequency due to the azimuthal component $B_{\varphi}$ to that due to the longitudinal component is

$\frac{m}{k R} \frac{B_{\varphi}}{(r / R) B_{z}}$

We computed the maximal value of the ratio for the model discussed in Sect. 2. We considered a weak azimuthal field and a relatively low value of $\alpha \ll 1$. To first order in $\alpha$ (small $\alpha$ approximation)

$$
\begin{aligned}
& B_{z} \simeq B_{z}(0), \\
& B_{\varphi}=\alpha B_{z}(0) f(x) / f_{\max } .
\end{aligned}
$$

Hence

$$
\frac{B_{\varphi}}{(r / R) B_{z}}=\alpha \frac{f(x)}{x} \frac{1}{f_{\max }} .
$$

For the prescription of $f(x)$ given by Eq. (8) we already know the value of $x_{\mathrm{m}}$ and $f_{\max }$. In addition, the function $f(x) / x$ is maximal at $x_{*}=\sqrt{p q}$, and $f\left(x_{*}\right) / x_{*}=p+q-2 \sqrt{p q}$. Hence the maximal value of

$$
\left.\frac{B_{\varphi}}{(r / R) B_{z}}\right|_{\max }=\alpha \frac{4}{(q-p)^{2}}(p+q-2 \sqrt{p q}) .
$$

For example, for the values $p=1 / 2$ and $q=3 / 2$ used in our calculations we find that

$$
\left.\frac{m}{k R} \frac{B_{\varphi}}{(r / R) B_{z}}\right|_{\max } \approx 1.08 m \frac{\alpha}{k R}
$$

As an example we consider a loop that is 10 times longer than the radius. For the fundamental mode along the tube axis it follows that $k R=\pi / 10$. So for $\alpha=1 / 10$ the ratio is about 0.34 . Thus, although the azimuthal component of the magnetic field is weak, its effect on the Alfvén frequency is substantial. For realistic loops it is a competition between the two quantities $k R$ and $\alpha$ which determine the ultimate effect.

We omitted resonantly damped eigenmodes. This choice restricts the maximal twist allowed in the system or conversely the allowed combinations of $\alpha$ and $k R$. Even for a constant density profile like the one considered in this work (see Eq. (1)), $\omega_{\mathrm{A}}$ is a function of position because $B_{\varphi}$ varies with position and the corresponding term is multiplied by the factor $1 / r$. A necessary (but not sufficient) condition for the absence of resonances is that $\max \omega_{\mathrm{Ai}}^{2} \leq \min \omega_{\mathrm{Ae}}^{2}$. This conditions is equivalent to

$\max \Phi<\pi\left(\sqrt{\rho_{\mathrm{i}} / \rho_{\mathrm{e}}}-1\right)$.

For $\rho_{\mathrm{i}} / \rho_{\mathrm{e}}=3$ this implies that $\max \Phi \lesssim 0.73 \pi$.

Condition (31) can be rewritten in terms of the function $f(x)$ as

$\frac{\alpha}{k R}<x_{*} \frac{f_{\max }}{f\left(x_{*}\right)}\left(\sqrt{\rho_{\mathrm{i}} / \rho_{\mathrm{e}}}-1\right)$.

For the typical values that we use in the computations the inequality is $\alpha / k R \lesssim 0.67$. So we can define the critical value of $\alpha$ as

$\alpha_{J}=k R x_{*} \frac{f_{\max }}{f\left(x_{*}\right)}\left(\sqrt{\rho_{\mathrm{i}} / \rho_{\mathrm{e}}}-1\right)$.

Then for values $\alpha>\alpha_{J}$ the azimuthal component of the magnetic field introduces resonances even in configurations with constant density. In addition to $\alpha_{J}$ we can consider the situation when the azimuthal component $B_{\varphi}$ dominates the variation of $\omega_{\mathrm{A}}^{2}$. For $m=1$ this happens when $\max \Phi(r) \geq \pi$ (see Eq. (25)). For the function $f(x)$ defined in Eq. (8) this can be reformulated into

$\frac{\alpha}{k R} \geq x_{*} \frac{f_{\max }}{f\left(x_{*}\right)}$.

This forces us to define a second critical value for $\alpha$

$\alpha_{M}=k R x_{*} \frac{f_{\max }}{f\left(x_{*}\right)}$,

which differers from $\alpha_{J}$ by the factor $\left(\sqrt{\rho_{\mathrm{i}} / \rho_{\mathrm{e}}}-1\right)$. For the present model with $p=1 / 2$ and $q=3 / 2, \alpha_{M} \simeq 1.09 \mathrm{kR}$. Below we used sufficiently low values of $\alpha$ compared to $\alpha_{J}$ and $\alpha_{M}$. This implies that even in the thin tube approximation we can use the small $\alpha$ approximation. 


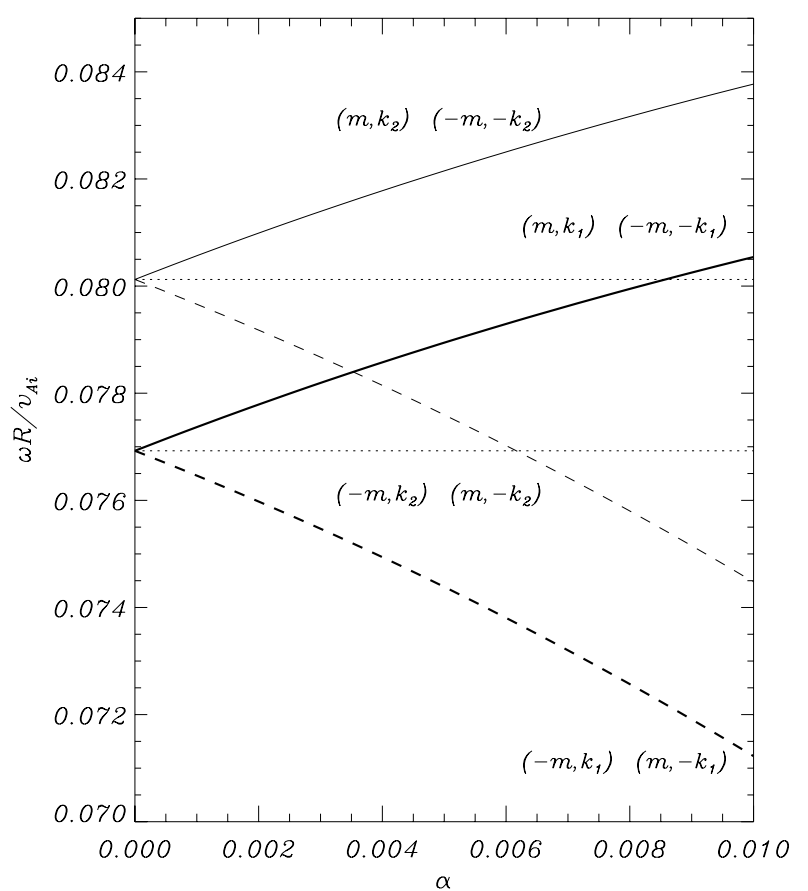

Fig. 1. Frequency as a function of twist $(\alpha)$. The curves are obtained by solving the eigenvalue problem in $1 \mathrm{D}$. The dotted line represents the solution for the untwisted case. For this particular example, $\rho_{\mathrm{i}} / \rho_{\mathrm{e}}=3$, $p=1 / 2, q=3 / 2$. The two sets of solutions are associated to $m=1$ and $k_{1}=\pi / 50 R$ (thick lines), and $k_{2}=\pi / 48 R$ (thin lines).

\section{Results: propagating waves}

We start by solving the eigenvalue problem for propagating waves using different pairs of the wavenumbers $m$ and $k$, which are assumed to be positive. We use a particular choice of parameters, $\rho_{\mathrm{i}} / \rho_{\mathrm{e}}=3, p=1 / 2, q=3 / 2$. In Fig. 1 the dependence of the eigenfrequency on $\alpha$, which measures the amount of twist, is plotted. For the pair $\left(m, k_{1}\right)$ the frequency is $\omega=k_{1} c_{\mathrm{k}}$ when $\alpha=0$, i.e., we recover the known result in the absence of twist (in the thin tube approximation), which is the kink speed

$c_{\mathrm{k}}=\sqrt{\frac{2}{1+\rho_{\mathrm{e}} / \rho_{\mathrm{i}}}} v_{\mathrm{Ai}}$,

where $v_{\mathrm{Ai}}=B_{0} / \sqrt{\mu_{0} \rho_{\mathrm{i}}}$ is the internal Alfvén speed for a straight field.

Figure 1 shows that the frequency increases monotonically with $\alpha$ (see continuous thick line). Exactly the same curve is obtained for the wavenumbers $\left(-m,-k_{1}\right)$, meaning that the results are degenerate with respect to a change in sign of the two wavenumbers. In constrast, for $\left(-m, k_{1}\right)$ and $\left(m,-k_{1}\right)$ the frequency decreases with $\alpha$ (see dashed thick line). This behaviour reflects the fact that, in contrast to the purely vertical magnetic field model, twist breaks the symmetry with respect to the propagation direction when the sign of one of the two wavenumbers is changed (see also the results of Vasheghani Farahani et al. 2010, for torsional Alfvén waves). The increase in frequency of the modes $\left(m, k_{1}\right)$ and $\left(-m,-k_{1}\right)$ with respect to the un-twisted case is around $4 \%$ while the decrease for $\left(-m, k_{1}\right)$ and $\left(m,-k_{1}\right)$ is $8 \%$ for $\alpha=0.01$. This means that a very weak twist (of about $1 \%$ ) produces an effect on the frequency that is not negligible. The maximum value of $\alpha$ in our calculations has been chosen to satisfy that $\alpha<\alpha_{J}$ and $\alpha<\alpha_{M}$ for the reasons explained in Sect. 3.2.

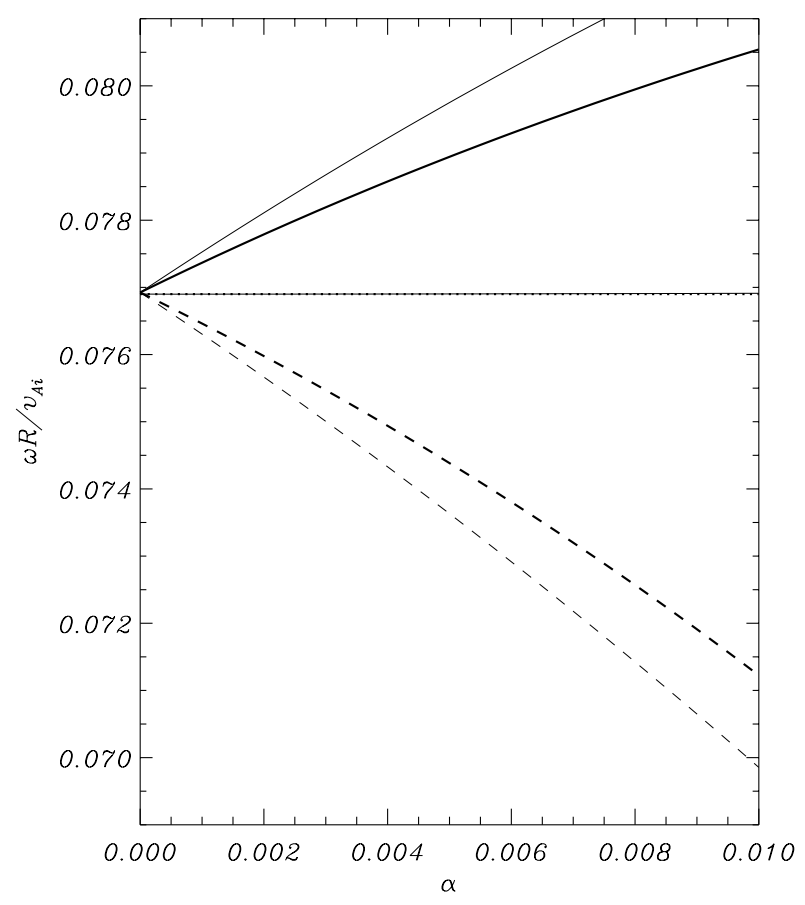

Fig. 2. Frequency as a function of twist $(\alpha)$. For this particular example, $\rho_{\mathrm{i}} / \rho_{\mathrm{e}}=3, p=1 / 2, q=3 / 2$ represented with thick lines while the case $p=0, q=2$ is plotted with thin lines. The dotted line represents the solution for the untwisted case. The horizontal continuous line is obtained by numerically solving the eigenvalue problem in $2 \mathrm{D}$. The same notation as in Fig. 1 is used.

Figure 1 also suggests that a linear variation of $\omega$ with $\alpha$ is a good approximation of the actual dependency,

$\omega \simeq k_{1} c_{\mathrm{k}}+a \alpha$, for $\left(m, k_{1}\right),\left(-m,-k_{1}\right)$,

$\omega \simeq k_{1} c_{\mathrm{k}}-a \alpha$, for $\left(m,-k_{1}\right),\left(-m, k_{1}\right)$,

$a$ being the slope of the linear approximation. The twist profiles considered by Goossens et al. (1992) and Ruderman (2007) are special since for those cases $a=0$, i.e., there is no frequency change for different wavenumbers (for example, $m= \pm 1$ ). However, for nonconstant twist configurations, like those studied here, there is always a frequency split.

Similar behaviour occurs for different longitudinal wave numbers, the curves are just shifted vertically (see continuous and dashed thin lines). The slope of the curves is independent of the value of $k$, as we can see in Fig. 1 (compare the curves for $k_{1}$ and $k_{2}$ ), but as we show below, it depends on the particular twist profile. According to Fig. 1 for a fixed frequency and fixed value of twist, there are always four possible solutions, $\left(m, k_{1}\right),\left(-m,-k_{1}\right),\left(-m, k_{2}\right),\left(m,-k_{2}\right)$ (see the intersection of the solid thick and dashed thin lines around $\omega=7.85 \times 10^{-2}$ and $\left.\alpha=3.5 \times 10^{-3}\right)$. This property will be used as the basis to construct approximate standing solutions in the following sections.

A qualitatively similar behaviour occurs when the parameters are changed. In Fig. 2 the results are plotted for $p=0$ and $q=2$ (see thin lines). Now the slope of the curves is steeper than for the case $p=1 / 2$ and $q=3 / 2$ but the curves still show a linear behaviour with $\alpha$. Since for the case $p=0$ and $q=2$ the region where the twist is localised is larger, the effect on the frequency is more pronounced. Indeed, we tested different nonconstant twist profiles and the curves are always linear with $\alpha$ or equivalently with $B_{\varphi}$ (for $\left|B_{\varphi}\right| \ll\left|B_{z}\right|$ ).

We now aim to know the effect of twist on the eigenfunctions. It can be shown from the MHD equations (see 
J. Terradas and M. Goossens: Transverse MHD waves in the presence of twist
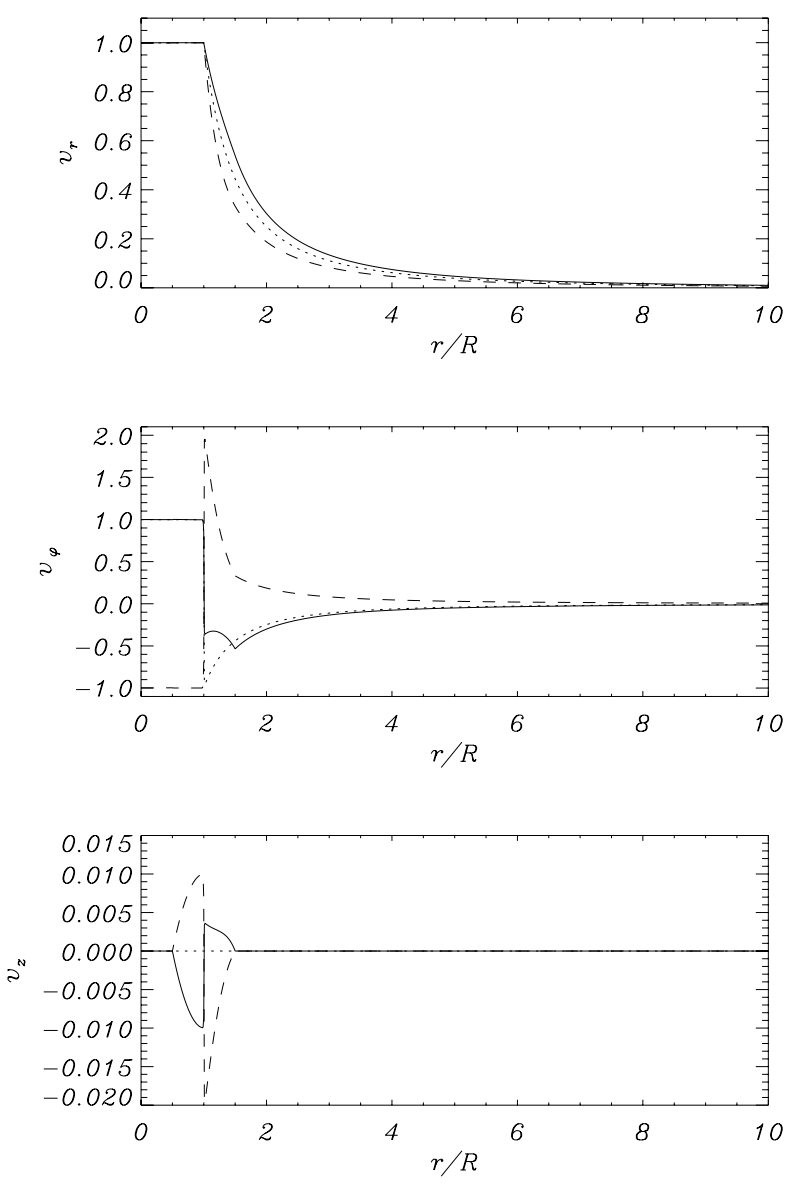

Fig. 3. Dependence of the three velocity components of the eigenfunction with the radial coordinate. For this particular example, $\rho_{\mathrm{i}} / \rho_{\mathrm{e}}=3$, $p=1 / 2, q=3 / 2$ and $\alpha=0.01$. The continuous and dashed lines correspond to the modes $(m, k)$ and $(-m, k)$, while the dotted line represents the mode $(m, k)$ in the absence of twist $(\alpha=0)$.

Appendix A) that if Fourier analysis is performed in both the azimuthal and longitudinal direction, like in the present case, then there is a phase shift between the radial component (pure real function) and the azimuthal and longitudinal components (purely imaginary functions). In Fig. 3 the radial dependence of $v_{r}, v_{\varphi}$ and $v_{z}$ is plotted for the modes $(m, k)$ (continuous line) and $(-m, k)$ (dashed line). The same figure is obtained for the modes $(-m,-k)$ and $(m,-k)$. We see that the radial dependence of the modes is essentially the same inside the tube and only in the external medium near the tube boundary the dependence is slightly different (compare also with the dotted line, which represents the eigenfunction for the classical untwisted tube). The azimuthal velocity component shows more relevant differences for the two modes. The mode $(m, k)$ has a reduced shear at the tube boundary while the opposite behaviour is displayed by the mode $(-m, k)$, showing an increased shear with respect to the untwisted case. This behaviour is also present in the $v_{z}$ component, which is introduced by the presence of twist. Since we are in the zero- $\beta$ approximation, the velocity along the magnetic field is zero, i.e., $v_{\|}=\boldsymbol{v} \cdot \boldsymbol{B}_{0} /\left|B_{0}\right|=0$, as expected. The $v_{z}$ component has a jump at the tube boundary and is different from zero only in the region where there is twist (in this particular example between $r=1 / 2 R$ and $r=3 / 2 R$ ). Notice that the amplitude of this velocity component is rather small in comparison with $v_{r}$ and $v_{\varphi}$ since we are considering a weak twist.

\section{Results: standing waves}

So far we focused on both azimuthally and longitudinally propagating waves. Our interest is now on the analysis of standing waves, if they exist in the twisted configuration.

\subsection{Standing in $\varphi$, propagating in $z$}

An azimuthally propagating wave, for example the mode $m=1$, produces a motion that displaces the whole tube and its axis is moving following a circular path because of the propagating nature of the wave in $\varphi$. If the motion is clockwise for $m$ then it is anti-clockwise for $-m$. For the tube to oscillate transversally, the propagating $m$ mode has to be combined with the $-m$ mode. When there is no twist, the frequency of the mode $(m, k)$ is the same as the for the mode $(-m, k)\left(\omega=k c_{\mathrm{k}}\right.$ in the thin tube limit), and the superposition is trivial. In the presence of twist the situation is more complicated since, as we have shown in Sect. 4, the modes $(m, k)$ and $(-m, k)$ have different frequencies. This means that with a single longitudinal wavenumber it is not possible to have a standing solution in the azimuthal direction. However, we have shown that we can always find another pair of wavenumbers with the same frequency. This means that the linear combination of the modes $\left(m, k_{1}\right)$ and $\left(-m, k_{2}\right)$ will lead to the standing solution in $\varphi$ we are looking for. Formally, we have to combine the two waves,

$y=f(r) \operatorname{expi}\left(\omega t+m \varphi+k_{1} z\right)+g(r) \operatorname{expi}\left(\omega t-m \varphi+k_{2} z\right)$

where $y$ is, for example, any of the velocity components of the eigenfunction. Note that $f(r)$ and $g(r)$ are different functions since they are associated to different pairs of wavenumbers $\left(m, k_{1}\right)$ and $\left(-m, k_{2}\right)$. However, in the thin tube approximation, when $k_{1} R \ll 1$ and $k_{2} R \ll 1$, the two functions are essentially the same $(f(r) \approx g(r)$ or $f(r) \approx-g(r)$ as Fig. 3 suggests $)$. This is a nontrivial assumption, which might not be a good approximation when we relax the thin tube and the small $\alpha$ approximations. Using this approximation, Eq. (39) reduces to

$y=f(r) \cos \left(m \varphi+k_{\mathrm{T}} z\right) \operatorname{expi}(\omega t+k z)$,

where we have introduced for convenience the following notation

$$
\begin{aligned}
k & =\frac{k_{1}+k_{2}}{2}, \\
k_{\mathrm{T}} & =\frac{k_{1}-k_{2}}{2} .
\end{aligned}
$$

If instead of the sum of the modes $\left(m, k_{1}\right)$ and $\left(-m, k_{2}\right)$ we choose the difference as the linear combination, we find

$y=f(r) \sin \left(m \varphi+k_{\mathrm{T}} z\right) \exp \mathrm{i}(\omega t+k z)$.

It is straightforward to construct the standing solution in $\varphi$ but propagating in the positive $z$-direction (note that Eq. (40) represents a wave propagating in the negative $z$-direction). The solution is based on the superposition of the modes $\left(-m,-k_{1}\right)$ and $\left(m,-k_{2}\right)$, and it is

$y=f(r) \cos \left(m \varphi+k_{\mathrm{T}} z\right) \exp \mathrm{i}(\omega t-k z)$.

Again the sinus solution is obtained when we subtract the modes.

Equations (40) and (44) represent propagating waves in the $z$-direction and produce a transverse displacement of the tube so that now it depends on $z$ through the term $k_{\mathrm{T}} z$. Thus, the direction of the transverse oscillation of the loop varies as the wave 
propagates along the tube. This represents a change in the polarisation of the transverse motion and is one of the main effects of twist on the eigenmodes. For the untwisted tube the polarisation of oscillation is always the same since $k_{\mathrm{T}}=0$.

The choice of $k_{1}$ and $k_{2}$ is not arbitrary since the modes with the pairs of wavenumbers $\left(m, k_{1}\right)$ and $\left(-m, k_{2}\right)$ (the same applies to the combination $\left(-m,-k_{1}\right)$ and $\left.\left(m,-k_{2}\right)\right)$ need to have the same frequency. The appropriate wavenumbers can always be determined numerically for a given frequency, as Fig. 1 suggests. However, if $\alpha$ is small, this selection is straightforward because we can use the approximations for the frequency (see Eqs. (37), (38)) to calculate $k_{1}$ and $k_{2}$,

$$
\begin{aligned}
& k_{1} \simeq \frac{\omega-a \alpha}{c_{\mathrm{k}}}, \\
& k_{2} \simeq \frac{\omega+a \alpha}{c_{\mathrm{k}}},
\end{aligned}
$$

where $a$ is the slope of the curves. Now using Eqs. (41) and (42) we have

$$
\begin{aligned}
k & \simeq \frac{\omega}{c_{\mathrm{k}}}, \\
k_{\mathrm{T}} & \simeq-\frac{a \alpha}{c_{\mathrm{k}}} .
\end{aligned}
$$

From the first equation we conclude that the dispersion relation of the wave in the twisted case, $\omega=k c_{\mathrm{k}}$, is exactly the same as in the untwisted tube (under the thin-tube approximation and for weak twist). Thus, for weak twist, there is no effect on the frequency of the propagating waves in $z$ and standing waves in $\varphi$. Nevertheless, from the second equation we find that the polarisation of the motion shows a dependence with twist $\left(k_{\mathrm{T}} \neq 0\right)$. This dependence is linear with $\alpha$. For the un-twisted case $(\alpha=0)$ we recover the well-known solution of the transverse kink mode without variation of polarisation along the tube.

\subsection{Standing in $z$, propagating in $\varphi$}

Now we seek solutions that are standing in the $z$-direction. These solutions have to satisfy line-tying conditions, i.e., the three velocity components must be zero at the footpoints of the loop, located at $z=0$ and $z=L$, where $L$ is the total length of the tube. We proceed as in the previous section and select the appropriate superposition of modes. A suitable choice is $\left(m, k_{1}\right)$ and $\left(m,-k_{2}\right)$,

$y=f(r)\left(\operatorname{expi}\left(\omega t+m \varphi+k_{1} z\right)+\operatorname{expi}\left(\omega t+m \varphi-k_{2} z\right)\right)$,

which can be written as

$y=f(r) \cos (k z) \exp \mathrm{i}\left(\omega t+m \varphi+k_{\mathrm{T}} z\right)$.

The other choice corresponds to the modes $\left(-m,-k_{1}\right)$ and $\left(-m,+k_{2}\right)$, which represent a propagating wave in the positive $\varphi$-direction,

$y=f(r) \cos (k z) \operatorname{expi}\left(\omega t-m \varphi-k_{\mathrm{T}} z\right)$.

As in the previous expressions, a sinus is obtained instead of a cosinus when the difference of the modes is considered instead of the sum,

$y=f(r) \sin (k z) \operatorname{expi}\left(\omega t+m \varphi+k_{\mathrm{T}} z\right)$,

and

$y=f(r) \sin (k z) \operatorname{expi}\left(\omega t-m \varphi-k_{\mathrm{T}} z\right)$.
Strictly speaking these modes cannot be classified as fully standing modes, because of the $z$ dependence in the exponential.

Applying line-tying conditions imposes restrictions on the wavenumbers. Using the sinus solution given by Eq. (52) or (53) allows us to easily impose that the velocity must be zero $z=$ 0 and $z=L$. The first condition is trivially satisfied while the second leads to

$\sin (k L)=0$,

and thus

$k=\frac{\pi}{L} n, n=1,2,3 \ldots$

This is essentially the same situation as in the untwisted tube, we have a discrete set of wavenumbers satisfying the boundary conditions.

In constructing the standing solution in $z$ we have assumed that this solution is the superposition of two propagating waves with the same frequency, different longitudinal wavenumbers and the same radial dependence. As we have explained, this is only true within limit $k R \ll 1$. In principle, we cannot assume a Fourier analysis in the $z$-direction because of line-tying conditions and consider single modes if we aim to obtain the full solution to the problem. For this reason, we now calculate the eigenmodes by solving the problem in $r$ and $z$ for a fixed $m$. This will allow us to compare the results with the semi-analytical results given by Eqs. (47) and (48). We used the two-dimensional version of the code PDE2D to calculate the modes with linetying conditions at $z=0$ and $z=L$. In Fig. 2 the eigenfrequency is plotted as a function of twist. Clearly, the frequency is almost independent of $\alpha$ (see the continuous horizontal line), which agrees with the analytical results obtained within the limit of small $\alpha$ given by Eq. (47) (compare also the continuous horizontal line with the dotted line, which corresponds to the untwisted case). Therefore, it is a good approximation (within the limit of small $\alpha$ ) to assume that we can construct standing eigenmodes by summing two propagating waves with the same frequency but slightly different wavenumbers.

A plot of the eigenfunction at $r=0$ and $\varphi=0$ as a function of $z$ is shown in Fig. 4. It is worth to mention that for the 2D problem the eigenfunction is always a complex number (this can be seen from Eqs. (A.1)-(A.6) in the Appendix) although the frequency is still a real magnitude since we do not consider unstable modes or resonances. For this reason we represent the modulus and the phase $(\delta)$ of the eigenfunction in Fig. 4. The modulus has a sinusoidal profile while the phase shows a linear dependence with $z$. This is exactly the behaviour predicted by Eq. (52). The slope of the phase is precisely the magnitude $k_{\mathrm{T}}$, which is negative according to Eq. (48). The $m=-1$ mode shows the expected positive slope, in agreement with Eqs. (53) and (48).

\subsection{Standing in $\varphi$ and $z$}

Once we have derived the solutions that are standing, or equivalent to standing, in one of the directions, it is straightforward to address the full standing problem. There are two ways to proceed that must be equivalent, either we add the solutions that are standing in $\varphi$ but propagating in $z$, or we combine the solutions that are (almost) standing in $z$ but propagating in $\varphi$. For example, from the combination of the solution given by Eq. (40), representing a standing wave in $\varphi$ but propagating in $z$, minus the complementary wave travelling in the opposite $z$-direction given by Eq. (44), we find the "full" standing solution

$y=f(r) \cos \left(m \varphi+k_{\mathrm{T}} z\right) \sin (k z) \exp (\mathrm{i} \omega t)$. 

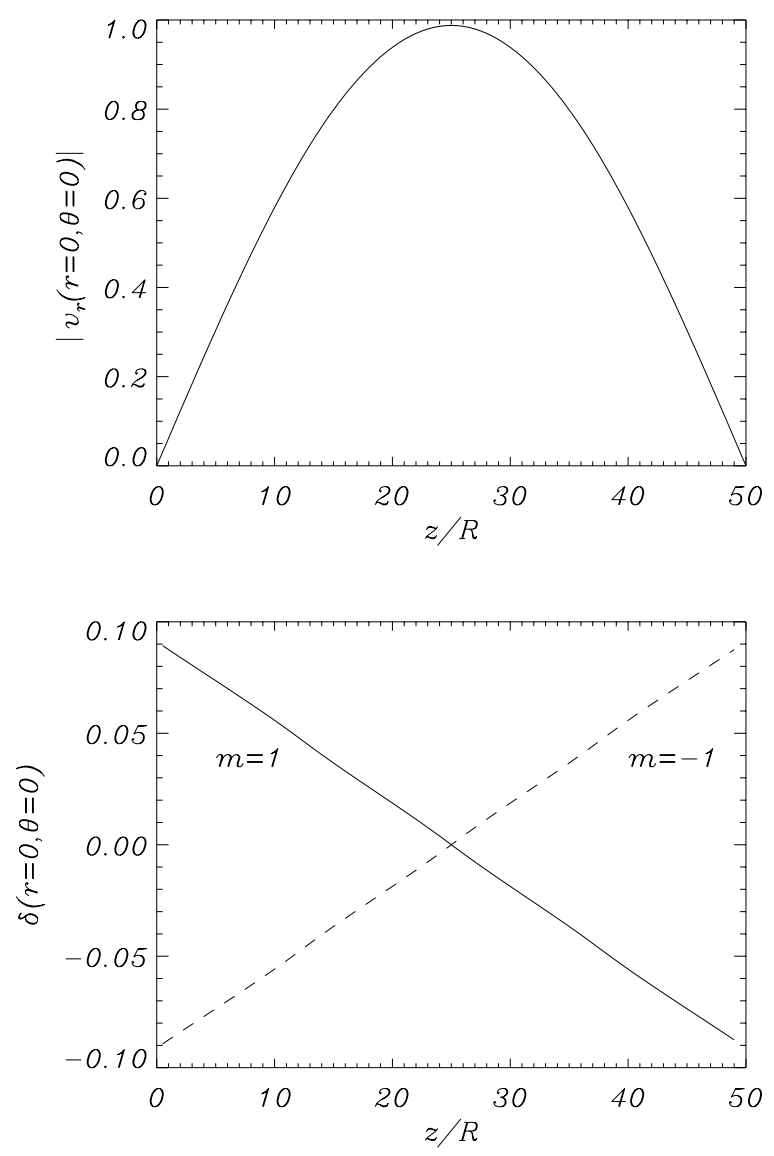

Fig. 4. Eigenfunction as a function of $z$ at the loop axis calculated numerically using line-tying conditions. The modulus and phase are plotted for the modes $m= \pm 1$.

It is easy to check that we obtain exactly the same expression by combining the solutions given in Eqs. (52), (53) for mainly representing standing waves in $z$ but propagating in $\varphi$.

Based on Eq. (56) and using the proper phase shifts between the components of the displacement, we obtain the following eigenfunction

$\xi_{r}=\tilde{f}(r) \cos \left(m \varphi+k_{\mathrm{T}} z\right) \sin (k z) \sin (\omega t)$,

$\xi_{\varphi}=\tilde{g}(r) \sin \left(m \varphi+k_{\mathrm{T}} z\right) \sin (k z) \sin (\omega t)$,

$\xi_{z}=\tilde{h}(r) \sin \left(m \varphi+k_{\mathrm{T}} z\right) \sin (k z) \sin (\omega t)$.

Similar expressions have been used in the past as approximate solutions to test the stability of different twisted magnetic configurations. However, as far as we know, these expressions have not been applied to stable kink waves.

From Eqs. (57)-(59) we can easily calculate the displacement of the tube axis, which is a relevant quantity when it comes to observations of transverse loop waves. According to Fig. 3, inside the tube $\tilde{f}(r) \simeq \tilde{g}(r)=$ const. in the thin-tube approximation, and the displacement in $z$ has a node at the axis. Thus, in Cartesian coordinates at the axis $(x=y=0)$

$\xi_{x}=\xi_{0} \cos \left(k_{\mathrm{T}} z+\varphi_{0}\right) \sin (k z) \sin (\omega t)$,

$\xi_{y}=\xi_{0} \sin \left(k_{\mathrm{T}} z+\varphi_{0}\right) \sin (k z) \sin (\omega t)$,

$\xi_{z}=0$,

$\xi_{0}$ being an arbitrary amplitude and $\varphi_{0}$ an arbitrary phase. These expressions show that the tube does not move along the axis while the displacement in the plane perpendicular to the axis

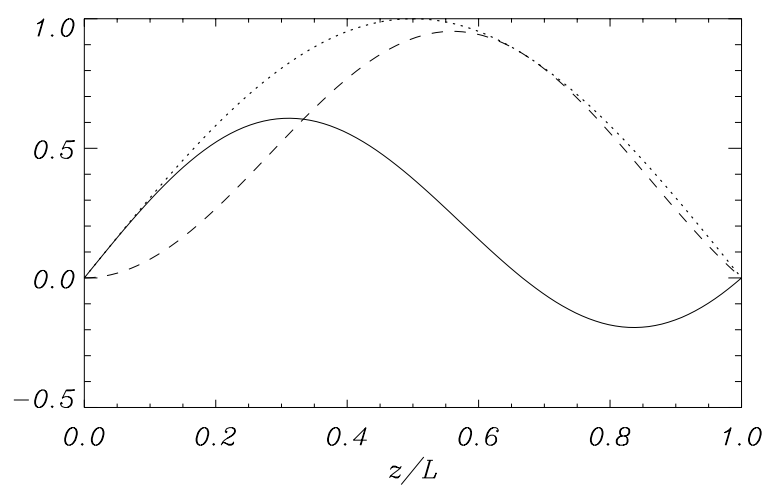

Fig. 5. Displacement of the tube axis as a function of position along the twisted tube. The continuous line corresponds to the displacement in the $x$-direction while the dashed line represents the displacement in the $y$-direction. The dotted line is the purely sinusoidal displacement in the $x$-direction for the untwisted case. In this example, $k_{\mathrm{T}}=-0.75 k$, $\varphi_{0}=0$, where $\xi_{0} / L=1$.

is given by the product of two sinusoidal terms, one involving a scale associated to the twist, $k_{\mathrm{T}}$, and another related to the longitudinal wavenumber, $k$. At the axis

$$
\sqrt{\xi_{x}^{2}+\xi_{y}^{2}+\xi_{z}^{2}}=\xi_{0} \sin (k z) \sin (\omega t),
$$

meaning that the total displacement is exactly the same as in the un-twisted case. The difference is in the components of the displacement which have a different weight depending on the position along the tube.

In Fig. 5 the displacement of the tube axis is plotted for a situation with moderate twist. Although the calculations in the previous sections were made using a very weak twist, we expect that for higher values of the $B_{\varphi}$ component the motion of the axis is qualitatively the same. The differences in the polarisation of the motion with respect to the untwisted situation (see dotted line) are evident in this plot. The motion of the tube is even more clear in Fig. 6 where the displacement of the tube is represented in three-dimensions at two different times during the oscillation. We clearly see the departures from the purely sinusoidal displacement for the untwisted case. In the top panel of Fig. 6 the displacement is closer to the first longitudinal harmonic than to the fundamental mode. However, at later stages of the oscillation, see bottom panel of Fig. 6, the motion resembles the fundamental mode. Thus, twist can have a pronounced effect on the displacement of the tube axis during the oscillation.

\section{Conclusions and discussion}

From inspection of the local Alfvén frequency it might be anticipated that an azimuthal magnetic field can have a relevant effect on MHD waves even if $\left|B_{\varphi}\right| \ll\left|B_{z}\right|$. It is the competition between the two quantities $k R$ and $\alpha=B_{\varphi, \max } / B_{z}(0)$ that determines the extent of the effect. In this work the quantity $\alpha$ is a small quantity by choice since we aim to focus on magnetic fields that are dominated by the longitudinal components. The quantity $k R$ is often small as most of the loops are much longer than wide or the longitudinal wavelength is much longer that the radius of the loop. So it is really a competition between two small quantities $k R$ and $\alpha$. In the present investigation we have deliberately excluded the regime $\alpha / k R \sim 1$ and focused our attention on $\alpha<\alpha_{J}$ and $\alpha<\alpha_{M}$. In this way we avoid the interesting complications of resonant absorption. However, restricting ourselves to these values of $\alpha$ means that the azimuthal magnetic fields are in a sense 

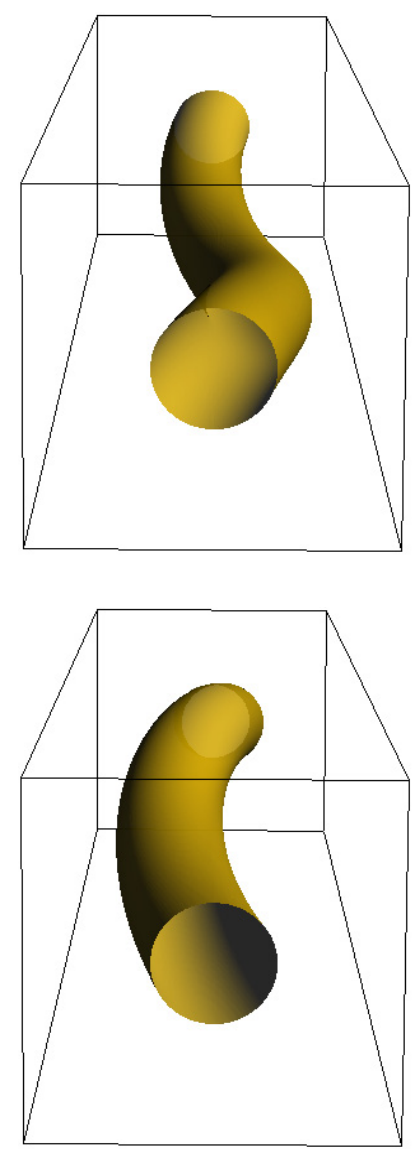

Fig. 6. Snapshots of the displacement of the tube with twisted magnetic field at two different times during one oscillation period. In this example, $k_{\mathrm{T}}=-0.75 k$. The $x$-axis is horizonal, the $y$-axis is vertical and the $z$-axis is perpendicular to the $x y$-plane. Line-tying conditions are applied at the front and back planes $(z=0$ and $z=L)$.

very weak, and that the effects on the MHD waves by azimuthal magnetic field are less pronounced than they effectively are.

In general, the frequencies of MHD waves in models with nonconstant magnetic twist are nondegenerate compared to their counterparts in models with constant magnetic twist. This means that the frequencies of propagating waves with wavenumbers $(m, k)$ and $(-m,-k)$ are equal but different from the frequency of the modes $(-m, k)$ and $(m,-k)$. Twist breaks the symmetry with respect to the propagation direction. This property has consequences for standing modes that require the superposition of propagating waves. We have shown, using an approximate method valid for weak twist, that the frequency of standing waves is unaffected by the presence of twist. Under such conditions the tube oscillates transversally at the characteristic kink frequency.

However, the most interesting effect of magnetic twist on standing oscillations is in the change of polarisation of the transverse motion along the tube. We have analytically demonstrated that the change in the direction of the transverse displacement of the tube is linearly proportional to the twist. Moreover, the polarisation of the displacement of the axis along the tube is simply described by the product of two sinusoidal terms involving a wavenumber associated to the twist, and a longitudinal wavenumber that satisfies the line-tying boundary conditions at the footpoints of the loop (this is very similar to the situation for a tube with a siphon flow along the axis studied by Terradas et al. 2011). For a very weak twist, for instance $1 / 10$ windings of the field along the loop axis, twist apparently introduces a motion perpendicular to the dominant direction of oscillation that is around nine times smaller in amplitude. The effect on the polarisation of the motion is small because the twist in the tube is very weak in this particular example. When the twist increases, the change in polarisation becomes stronger, as is shown in Figs. 5 and 6. An important result here is that a weak twist can produce displacements in any direction perpendicular to the unperturbed tube axis. Thus, in contrast to the effect of curvature of the magnetic field lines and the effect of nonuniform cross section, which both introduce vertical and horizontal modes, twist produces displacements in all directions. This agrees with the results of Ruderman \& Scott (2011), who considered kink oscillation of a nonplanar coronal loop involving magnetic twist. In view of these results the role of twist on stable transverse motions has been underestimated in the context of loop oscillations. Potentially, the signatures of twist on standing transverse oscillations could be used as a way to infer the value of the azimuthal component of the magnetic field in coronal loops. This could have clear seismological applications. However, the fact that real coronal loops are in many cases nonplanar and noncircular, i.e., they have an helical shape (see the review of Aschwanden 2009), might complicate the comparison between theory and observations.

Finally, it is worth to notice that the analysis performed in this paper is based on the assumption of very weak twist. This has allowed us to avoid some complications that appear when the $B_{\varphi}$ component of the magnetic field is increased. First of all, Alfvénic resonances cannot be avoided for moderate twist (see the work of Karami \& Bahari 2010). Second, the modes might become kink-unstable. A detailed investigation of these effects on standing kink modes need to be addressed in future works.

Acknowledgements. J.T. acknowledges support from the Spanish Ministerio de Educación y Ciencia through a Ramón y Cajal grant. Funding provided under the project AYA2011-22846 by the Spanish MICINN and FEDER Funds, and the financial support from CAIB through the "Grups Competitius" scheme and FEDER Funds is also acknowledged by J.T. M.G. acknowledges financial support received during his visits at Universitat de les Illes Balears (UIB), funding under grant AYA2011-22846 and University of Leuven GOA 2009/009 is also acknowledged. The authors also thank J. Andries for his comments and suggestions during the preparation of this paper.

\section{Appendix A}

The ideal linearised MHD equations in the zero- $\beta$ regime in the presence of magnetic twist are the following:

$$
\begin{aligned}
\mathrm{i} \omega v_{r}= & \frac{1}{\mu_{0} \rho}\left(\left(\frac{\partial b_{r}}{\partial z}-\frac{\partial b_{z}}{\partial r}\right) B_{z}-\frac{\mathrm{d} B_{z}}{\mathrm{~d} r} b_{z}\right. \\
& \left.-\frac{1}{r}\left(\frac{\partial r b_{\varphi}}{\partial r}-\mathrm{i} m b_{r}\right) B_{\varphi}-\frac{1}{r} \frac{\mathrm{d}\left(r B_{\varphi}\right)}{\mathrm{d} r} b_{\varphi}\right) \\
\mathrm{i} \omega v_{\varphi}= & \frac{1}{\mu_{0} \rho}\left(-\left(\frac{\mathrm{i} m}{r} b_{z}-\frac{\partial b_{\varphi}}{\partial z}\right) B_{z}+\frac{1}{r} \frac{\mathrm{d}\left(r B_{\varphi}\right)}{\mathrm{d} r} b_{r}\right) \\
\mathrm{i} \omega v_{z}= & \frac{1}{\mu_{0} \rho}\left(\left(\frac{\mathrm{i} m}{r} b_{z}-\frac{\partial b_{\varphi}}{\partial z}\right) B_{\varphi}+\frac{\mathrm{d} B_{z}}{\mathrm{~d} r} b_{r}\right) \\
\mathrm{i} \omega b_{r}= & B_{z} \frac{\partial v_{r}}{\partial z}+B_{\varphi} \frac{\mathrm{i} m}{r} v_{r}, \\
\mathrm{i} \omega b_{\varphi}= & B_{z} \frac{\partial v_{\varphi}}{\partial z}-B_{\varphi} \frac{\partial v_{z}}{\partial z}-B_{\varphi} \frac{\partial v_{r}}{\partial r}-v_{r} \frac{\mathrm{d} B_{\varphi}}{\mathrm{d} r} \\
\mathrm{i} \omega b_{z}= & \frac{1}{r}\left(-B_{z} \frac{\partial r v_{r}}{\partial r}-r v_{r} \frac{\mathrm{d} B_{z}}{\mathrm{~d} r}-B_{z} \mathrm{i} m v_{\varphi}+B_{\varphi} \mathrm{i} m v_{z}\right)
\end{aligned}
$$


where $\boldsymbol{v}=\left(v_{r}, v_{\varphi}, v_{z}\right)$ is the velocity and $\boldsymbol{b}=\left(b_{r}, b_{\varphi}, b_{z}\right)$ is the perturbed magnetic field. If a Fourier analysis is performed in the $z$-direction, the derivatives with respect to $z$ have to be replaced by $i k$.

\section{References}

Aschwanden, M. J. 2009, Space Sci. Rev., 149, 31

Aschwanden, M. J., \& Schrijver, C. J. 2011, ApJ, 736, 102

Aschwanden, M. J., Fletcher, L., Schrijver, C. J., \& Alexander, D. 1999, ApJ, 520,880

Bennett, K., Roberts, B., \& Narain, U. 1999, Sol. Phys., 185, 41

Brown, D. S., Nightingale, R. W., Alexander, D., et al. 2003, Sol. Phys., 216, 79

Carter, B. K., \& Erdélyi, R. 2008, A\&A, 481, 239

Díaz, A. J., Oliver, R., Ballester, J. L., \& Soler, R. 2011, A\&A, 533, A95

Einaudi, G., \& van Hoven, G. 1983, Sol. Phys., 88, 163

Erdélyi, R., \& Carter, B. K. 2006, A\&A, 455, 361

Erdélyi, R., \& Fedun, V. 2006, Sol. Phys., 238, 41

Erdélyi, R., \& Fedun, V. 2007, Sol. Phys., 246, 101

Erdélyi, R., \& Fedun, V. 2010, Sol. Phys., 263, 63

Giachetti, R., van Hoven, G., \& Chiuderi, C. 1977, Sol. Phys., 55, 371

Goossens, M., Hollweg, J. V., \& Sakurai, T. 1992, Sol. Phys., 138, 233

Hood, A. W., \& Priest, E. R. 1979, Sol. Phys., 64, 303

Hood, A. W., \& Priest, E. R. 1981, Geophys. Astrophys. Fluid Dyn., 17, 297
Hood, A. W., Priest, E. R., \& Einaudi, G. 1982, Geophys. Astrophys. Fluid Dyn., 20,247

Hood, A. W., Archontis, V., Galsgaard, K., \& Moreno-Insertis, F. 2009, A\&A, 503, 999

Karami, K., \& Bahari, K. 2010, Sol. Phys., 263, 87

Kruskal, M. D., Johnson, J. L., Gottlieb, M. B., \& Goldman, L. M. 1958, Phys. Fluids, 1, 421

Moreno-Insertis, F., \& Emonet, T. 1996, ApJ, 472, L53

Nakariakov, V. M., Ofman, L., Deluca, E. E., Roberts, B., \& Davila, J. M. 1999, Science, 285, 862

Priest, E. R., Hood, A. W., \& Anzer, U. 1989, ApJ, 344, 1010

Raadu, M. A. 1972, Sol. Phys., 22, 425

Ruderman, M. S. 2007, Sol. Phys., 246, 119

Ruderman, M. S., \& Erdélyi, R. 2009, Space Sci. Rev., 149, 199

Ruderman, M. S., \& Scott, A. 2011, A\&A, 529, A33

Sewell, G. 2005, The Numerical Solution of Ordinary and Partial Differential Equations, 2nd edn. (Newark, NJ: Wiley)

Shafranov, V. D. 1957, J. Nucl. Energy II, 5, 86

Suydam, B. R. 1958, Proc-1958-UN, 187

Terradas, J., Oliver, R., \& Ballester, J. L. 2006, ApJ, 642, 533

Terradas, J., Arregui, I., Verth, G., \& Goossens, M. 2011, ApJ, 729, L22

Vasheghani Farahani, S., Nakariakov, V. M., \& van Doorsselaere, T. 2010, A\&A, 517, A29

Velli, M., Hood, A. W., \& Einaudi, G. 1990, ApJ, 350, 428

Zaqarashvili, T. V., Díaz, A. J., Oliver, R., \& Ballester, J. L. 2010, A\&A, 516, A84 